\title{
An extensive $\Delta a$-photometric survey of southern B and A type bright stars $^{\star, \star \star}$
}

\author{
N. Vogt ${ }^{1, \star \star \star}$, F. Kerschbaum ${ }^{2}$, H.M. Maitzen ${ }^{2}$, and M. Faúndez-Abans ${ }^{3}$ \\ 1 Departamento de Astronomía y Astrofísica, Pontificia Universidad Católica de Chile, Casilla 104, Santiago 22, Chile \\ ${ }^{2}$ Institut für Astronomie der Universität Wien, Türkenschanzstraße 17, A-1180 Wien, Austria \\ 3 CNPq/Laboratório Nacional de Astrofísica, Caixa Postal 21, CEP 37500-000 Itajubá, MG, Brasil
}

Received July 31, 1997; accepted January 16, 1998

\begin{abstract}
Photoelectric photometry of 803 southern BS objects in the $\Delta a$-system as detection tool for magnetic chemically peculiar $(=\mathrm{CP} 2)$ stars has been carried out and compared to published spectral types. The statistical yield of such objects detected by both techniques is practically the same. We show that there are several factors which contaminate the search for these stars, but this contamination is only of the order of $10 \%$ in both techniques. We find a smooth transition from normal to peculiar stars.

Our sample exhibits the largest fraction of CP2 stars at their bluest colour interval, i.e. $10 \%$ of all stars in the colour range $-0.19 \leq B-V<-0.10$ or $-0.10 \leq b-y<$ -0.05 . No peculiar stars based on the $\Delta a$-criterion were found at bluer colours. Towards the red side the fraction of CP2 stars drops to about $3 \%$ for positive values of $B-V$ or $b-y$ with red limits roughly corresponding to normal stars of spectral type A5.

The photometric behaviour of other peculiar stars: Am, HgMn, $\delta$ Del, $\lambda$ Boo, He abnormal stars, as well as $\mathrm{Be} /$ shell stars and supergiants shows some slight, but definite deviations from normal stars. Spectroscopic and visual binaries are not distinguished from normal stars in their $\Delta a$ behaviour.

The results of this work justify larger statistical work (e.g. in open clusters) employing more time-saving photometric methods (CCD).
\end{abstract}

Send offprint requests to: H.M. Maitzen, e-mail: MAITZEN@ASTRO.AST.UNIVIE.AC.AT

* Based on observations obtained at the European Southern Observatory, La Silla, Chile. This research has made use of the Simbad database, operated at CDS, Strasbourg, France.

$\star \star$ Table 2 is only available in electronic form via anonymous ftp 130.79.128.5 or http://cdsweb.u-strasbg.fr/Abstract.html $\star \star \star$ On leave: Sternwarte Sonneberg, Sternwartestrasse 32, D96515 Sonneberg, Germany.
Key words: techniques: photometric — stars: chemically peculiar - catalogues

\section{Introduction}

Statistical studies on chemically peculiar stars of the upper main sequence (CP-stars) have been primarily based on spectroscopic detections. However, in the case of the magnetic subgroup CP2 (and the magnetic part of subgroup CP4, the Helium-weak stars) use was made of their broad band $520 \mathrm{~nm}$ flux depression to develop photometric indices in the Geneva system $(\Delta(V 1-G)$, Hauck 1974) and in the system of Maitzen $(1976, \Delta a)$ in order to detect those objects in larger samples and in an impersonal way.

The identification power of $\Delta a$ was demonstrated by Maitzen \& Vogt (1983) for a sample of 339 southern CP2 stars detected spectroscopically by Bidelman \& MacConnell (1973). Especially for the most numerous subgroup, the Silicon stars, the detection capacity of the $\Delta a$ index approaches $100 \%$.

After having shown that virtually all spectroscopically detected CP2 stars are also identified by the $\Delta a$ index as peculiar, the second task to embark on has been to show that systematically positive $\Delta a$-values are only found and typical for magnetic CP-stars. In this process the case of Pleione (Maitzen \& Pavlovski 1987), a B8 star with emission and shell episodes introduced a new aspect: it had a distinctly positive $\Delta a$-value in 1984 which faded to zero with fading shell phase. This means that a certain impurity for the magnetic $\mathrm{CP}$ detection capability of $\Delta a$ may have to be taken into account for stars similar to Pleione, hence the Be/shell stars. A current programme (Maitzen \& Pavlovski 1997), however, is showing that this impurity is of minor importance since the case of Pleione is a very outstanding one, and that $\Delta a$ values among $\mathrm{Be} /$ shell stars are only marginally positive. It is worthwile mentioning at 
the same time, that those stars while in the emission phase usually show a systematically negative $\Delta a$-index.

Our present investigation which comprises the largest sample of programme stars ever measured in $\Delta a$, i.e. 803 objects, aims at:

1. solving the problem whether $\Delta a$-detections match the existing spectroscopic detections of magnetic CP-stars,

2 . finding out whether there are $\Delta a$ peculiar stars with no published spectroscopic evidence of peculiarity,

3. studying the transition from normal to peculiar stars in terms of the $\Delta a$-index,

4. identifying systematic $\Delta a$-behaviour, both positive and negative for other groups of stars (e.g. Am=CP1 stars, $\lambda$ Boo stars, Be and B shell stars).

Concerning spectroscopic evidence we were hampered by the following situation: although the photometric material had been gathered about two decades ago, at that time its spectroscopic counterpart was not mature for reasonable comparison, since the spectral classifications in the old BS-catalogue were based on both very inhomogeneous material and classificators. The situation has improved due to revised spectral types in the new BS-catalogue (Hoffleit 1982), but even more so by the huge undertaking of the Michigan catalogue series started nearly two decades ago, which stands out by its homogeneous plate material and uniform classification skill (Houk \& Cowley 1975; Houk 1978; Houk 1982; and Houk \& Smith-Moore 1988). Though it has not been fully finished yet, we deem it justified now to proceed with the publication of our comparisons.

\section{Observations and reduction}

\subsection{The sample}

Our sample comprises to a high degree of completeness $(90 \%)$ all stars in the Bright Star Catalogue fainter than $V=5.1$ within the spectral domain B0 to A9, in the R.A. range $23-8$ hours and south of declination $10^{\circ}$ which corresponds to the concept of "southern stars" used in the title of this paper. Occasionally, brighter stars have been included, as well as stars with later spectral types (up to F5) and slightly outside the right ascension domain indicated.

Figure 1 shows the distribution in galactic coordinates of the 803 stars of this sample. Since the great majority lies in the third galactic quadrant which is nearly empty of interstellar dust out to 500 pc (Lucke 1978), reddening plays an unimportant role for main sequence $\mathrm{A}$ type stars, but has to be accounted for in the case of B type stars (Crawford-calibration and Q-method) and A-F supergiants (intrinsic colours corresponding to spectral types). We used dereddened values $(b-y)_{0}$ based on the Stroemgren-data compiled by Hauck \& Mermilliod (1980).

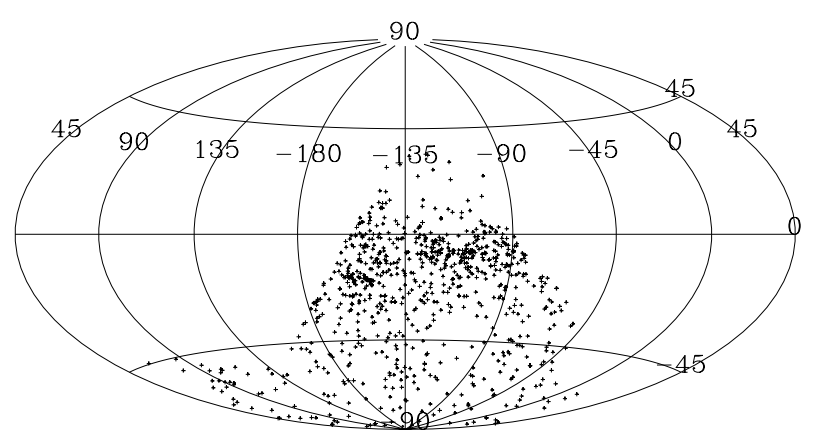

Fig. 1. Distribution of the observed Bright Stars in galactic coordinates

\subsection{Observing runs}

The measurements in the 3 filter system $(g 1, g 2, y)$ were obtained at the ESO $50 \mathrm{~cm}$ telescope on La Silla by two of us (NV, MFA) with the assistance of Messrs. Alberto Blest, Joaquín Perez and Gorki Román. Contributions to this programme were made on 59 nights between September 10, 1974 and June 30, 1976 (Table 1 in the Appendix). The instrument configuration was the same as described in Maitzen \& Vogt (1983) and their filter set of system No. 2 was used.

The majority of stars were observed only once $(86 \%)$ and $11 \%$ had 2 measurements. The remaining $3 \%$ were measured 3 and 4 times and served to determine the zeropoint shifts both in $a$ and $g 1-y$ between different observing runs.

The results of our photometry together with pertinent published data are contained in Table 2 in the Appendix.

\subsection{Line of normality $\left.a_{0}\left[(b-y)_{0}\right)\right]$}

In order to minimize the influence of non-normal stars on the determination of the locus of normal stars in the $a$ vs. $(b-y)_{0}$ diagram we eliminated all stars with known peculiar types and emission line stars from our sample, using spectroscopic data from the BS and Michigan catalogues. Additionally, in order to avoid uncontrolled complexity we decided to exclude spectroscopic binaries and visual binaries with separations of less than 15 arcseconds and magnitude differences of less than 3 . This way 476 objects remained as sample of normal stars.

A second order regression yielded the normality line: $a_{0}=593.3+0.08496(b-y)_{0}-7.082^{-5}(b-y)_{0}^{2}[\mathrm{mmag}] .(1)$ The standard deviation around this line is $4.74 \mathrm{mmag}$. The sample of normal stars together with the normality line and $3 \sigma$ lines above and below it are displayed in Fig. 2 .

Considering only stars with $(b-y)_{0}$ bluer than 0.1 the standard deviation drops to $4.5 \mathrm{mmag}$ whence we shall register all $\Delta a$-values with $14 \mathrm{mmag}$ and more as peculiar in this range. The scatter around the normality line increases to $5.6 \mathrm{mmag}$ for the remaining stars. Since this 


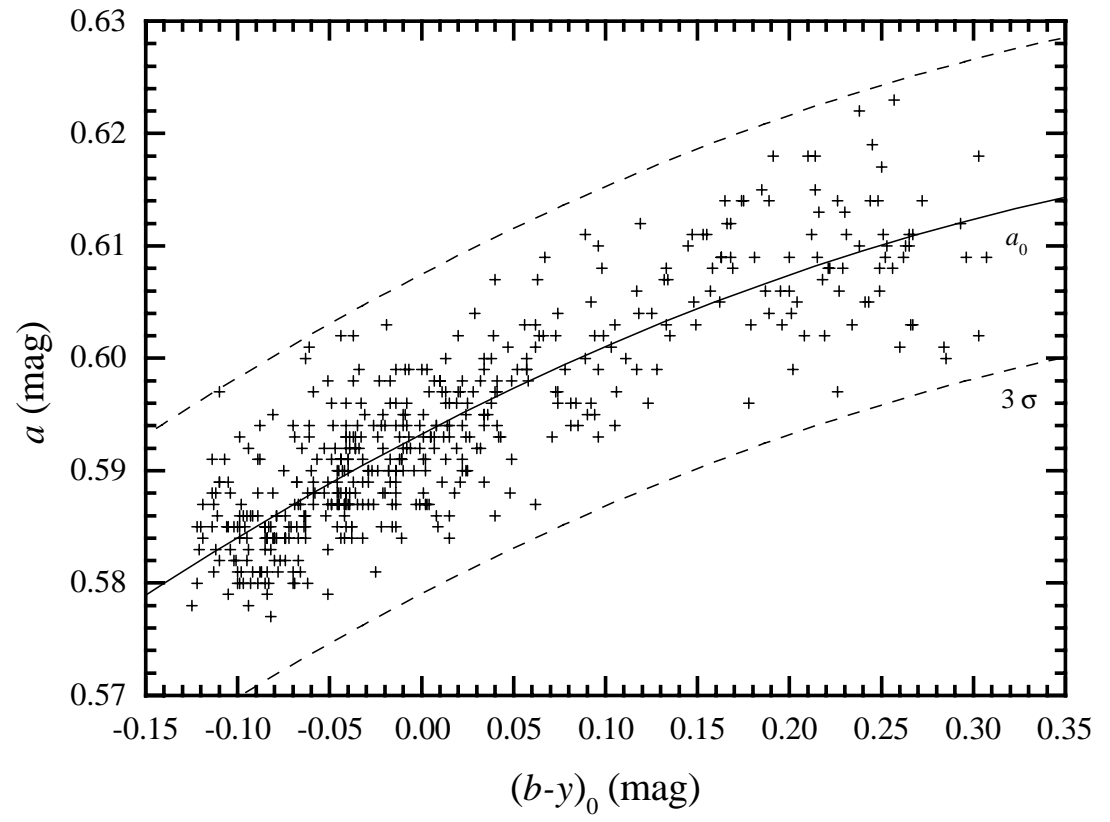

Fig. 2. Normal stars and the normality line

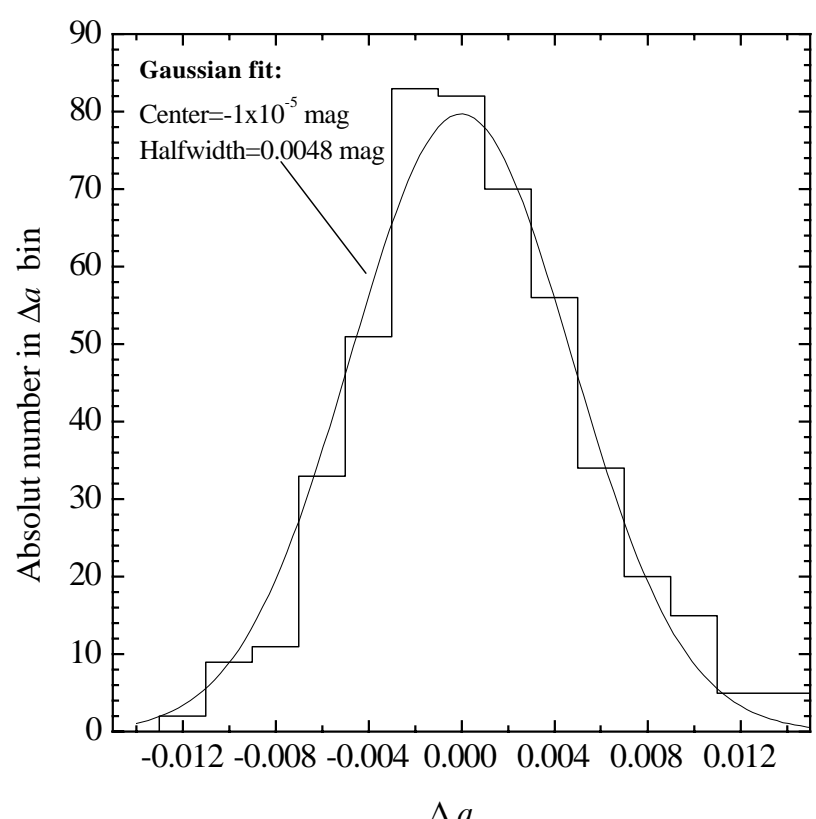

Fig. 3. Histogram of $\Delta a$ values for normal stars

is not to be attributed to random effects (the stars having the same limiting magnitude as the foregoing ones) it could be explained by intrinsic metallicity differences among the cooler stars of our sample.

Figure 3 shows the histogram of deviations $\Delta a=a-$ $a_{0}$ with a binning of $2 \mathrm{mmag}$. It is interesting to notice that the distribution of the positive $\Delta a$-values declines somewhat less steeply from the maximum than for the negative ones. This could be explained by the existence of marginally peculiar stars which escape detection by both the photometric $3 \sigma$ criterion and classification dispersion spectroscopy and form a transition population between peculiar and normal stars. Hints for that can be found e.g. in Maitzen \& Vogt (1983).

\subsection{The $g 1-y$ colour index}

Measurements in the 3 filter system $g 1, g 2, y$ yield not only the index $a$, but also colour differences one of which is $g 1-y$ with the longest baseline in wavelength. It is interesting to ask, especially with such a large sample of objects, both normal and peculiar, whether it shows a good correlation with $b-y$ normally used as reference colour when determining $\Delta a$-values. If so, it might be safely used instead of $b-y$, especially if the latter should not be available.

Figure 4 shows the diagram $g 1-y$ versus $b-y$ with the linear regression:

$g 1-y=490.5+0.6022(b-y)[\mathrm{mmag}]$.

The average scatter around this line is $6.51 \mathrm{mmag}$ and the correlation coefficient 0.9946 . Considering that the errors in the abscissa values $b-y$ are of the same order, the very high degree of correlation becomes obvious. This is not straightforward, since also peculiar stars were included, and some differentiation between the bandpasses of $b$ and $g 1$ could be expected. Moreover, no dereddening procedure was applied. If one looks carefully, one will notice a slight preponderance of points above the regression line in the interval $0.050 \leq b-y<0.150$, hence among the mid A type stars. This effect which is on the average smaller than 0.010 mag might be due to strong Fe I lines in the passband of $g 1$ and the rather high percentage of metallic line stars in this spectral region. 


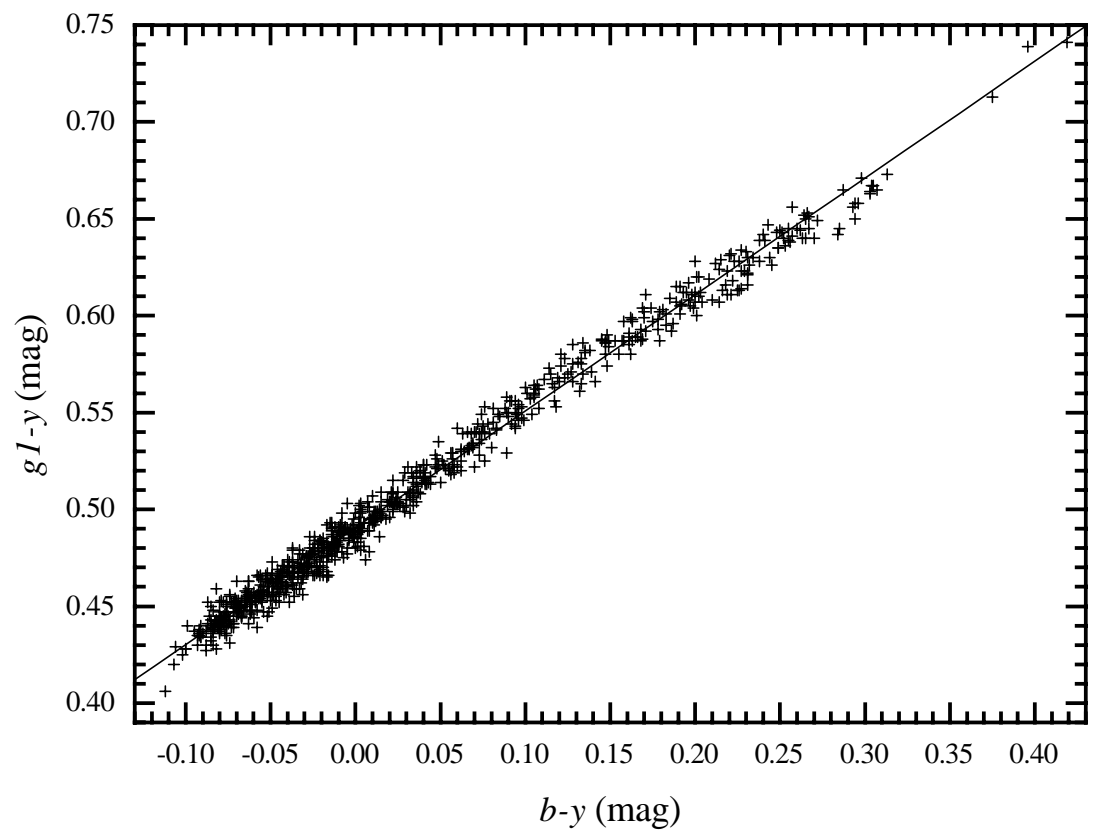

Fig. 4. Correlation of the $g 1-y$ and the $b-y$ colour indices

\section{Discussion}

Figure 5 contains the $a$ vs. $(b-y)_{0}$ diagram for the stars not contained in the sample of normal stars defined in 2.3 and displayed in Fig. 2. Not included, however, are here the normal double stars which are shown in Fig. 7. Different symbols denote the stellar types represented here including $\mathrm{CP} 1, \mathrm{CP} 2, \mathrm{CP} 3, \mathrm{CP} 4, \delta$ Del, $\lambda$ Boo, emission line, supergiant stars and the Barium object. The reference line of normal stars obtained in Fig. 2 and the positive and negative $3 \sigma$ lines have been drawn in order to visualize the more or less deviating photometric behaviour of the different groups discussed in Sect. 3.5.

\subsection{Stars more than $3 \sigma$ above the $a_{0}$-line}

First, we discuss stars which lie above the normality line by at least $3 \sigma$ (see Sect. 2.3) dividing them in three groups according to their published spectral peculiarity:

\subsubsection{Most certain CP2-stars}

They are classified as such in the BS and, if contained, in the Michigan catalogues; we recognize 20 objects 13 of which show characteristics of the hotter group (Silicon stars), 7 are cooler CP2-stars.

\subsubsection{Certain CP2-stars}

Those objects have discrepant peculiarity assignment in BS and Michigan. They are altogether 6 objects, 5 belonging to the Silicon group, and one is He weak (CP4).

It is remarkable that Michigan peculiarity coincides in 4 cases with peculiar $\Delta a$, the BS Catalogue in 2.

\subsubsection{Moderate peculiar $\Delta a$-cases}

Those 7 objects have neither a CP2-assignment in BS nor Michigan. They can be divided as follows:

Four bona fide CP2/4 stars: HR 1754 has been classified as B7p-He weak Silicon star $(=\mathrm{CP} 4)$ by Abt \& Cardona (1983). We have additional $\Delta a$-photometry for this star and its brighter companion HR 1753 obtained by one of us (H.M.M.) at the $1 \mathrm{~m}$ ESO-telescope on La Silla from Dec. 28, 1988 to Jan. 1, 1989 on five nights. The mean $\Delta a$ values of both stars, 0.020 and $-0.002 \mathrm{mag}$, resp., agree perfectly with our present results and are supported by $\Delta(V 1-G)=0.011$ derived from Rufener (1988). Thus HR 1754 belongs to the magnetic branch of CP4 stars (see Maitzen 1981 and 1984) which is also corroborated by the photometric variation with rotational period $P=2.287$ days (Catalano et al. 1991).

HR 1973 with the second largest $\Delta a(=0.023 \mathrm{mag})$ in this group has rather high $m_{1}$-values in the literature $(0.129,0.152,0.163 \mathrm{mag})$ considering its early spectral type and negative $b-y$. Calculating $\Delta(V 1-G)=0.011$ from Rufener (1988) we find support for photometric CP2behaviour. One should investigate the spectroscopic characteristics of this object in more detail in order to find the reason for its undetected peculiarity (spectral line variability may hamper detection of peculiarity, see Maitzen \& Lebzelter 1993).

HR 2424. It was measured already by Maitzen \& Vogt (1983) yielding exactly the same result: $\Delta a=0.014$. Since Buscombe (1980) gives B9pSi and Hauck \& North (1982) obtain also photometric peculiarity in the Geneva system this star can be safely assumed to be of CP2 type. 


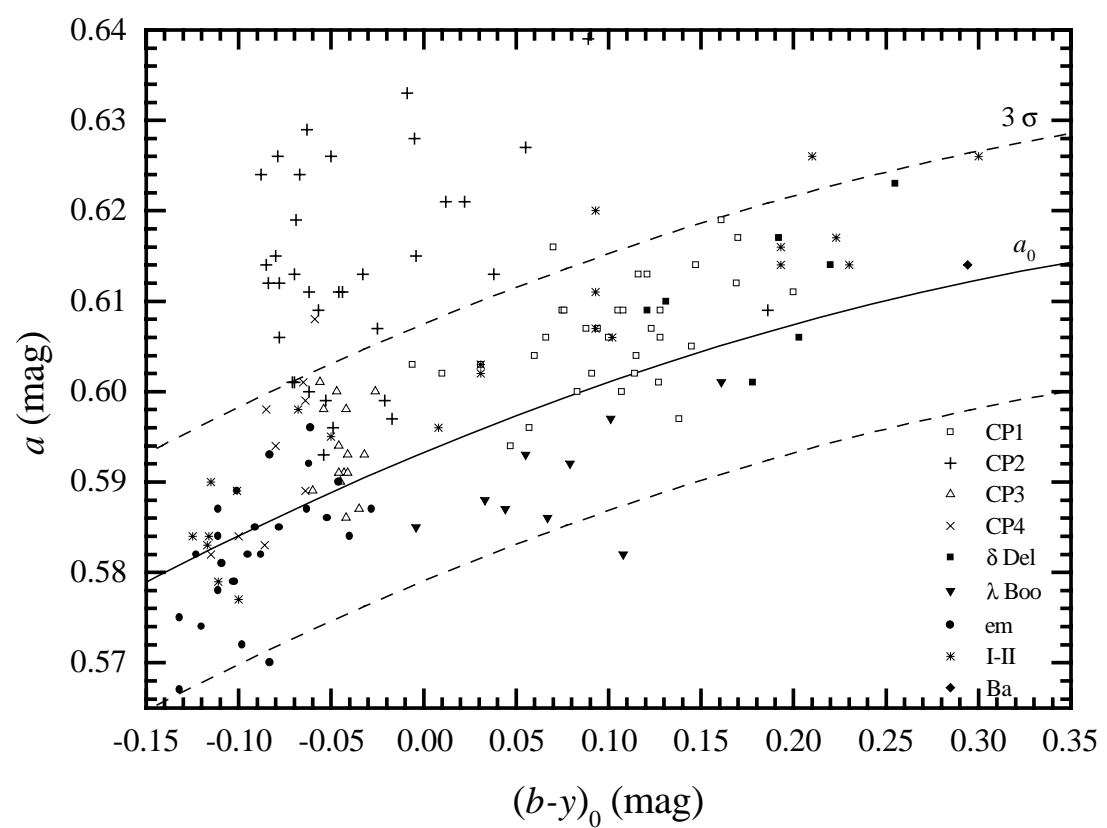

Fig. 5. $a$ versus $(b-y)_{0}$ diagram for peculiar stars

HR 3203. This star appears as supergiant in both catalogues and would therefore not belong to the CP2/4 class. However, its Geneva colours contradict supergiant luminosity ( $\log g=3.4$ ) and support peculiarity through $\Delta(V 1-G)=0.011$. As it is not an infrequent case that Michigan classifies high luminosity where other sources have low luminosity (in agreement with photometry) we tend to attribute peculiarity to this star which should of course be substantiated by new spectroscopy.

One Am (=CP1) star: HR 1730, while classified A2 III/IV in the Michigan catalogue (and repeated in the BS catalogue), has an entry in the catalogue of Am stars by Curchod \& Hauck (1979). This object represents the strongest positive deviation $(\Delta a=0.017)$ of the Am-stars discussed in Sect. 3.5.3.

Two A-F supergiants: HR 2785 and HR 2874. Both had to be dereddened taking into account their spectral types. In spite of the related uncertainty it is impossible to deny a substantial positive deviation from the normality line which however occurs in the spectral domain of the late A-type stars, where CP2-stars seldom lie above this line.

\subsection{Stars with solely spectroscopic peculiarities}

In the second part it is to be discussed which objects apparently are CP2 according to some spectroscopic sources, but lack photometric peculiarity (i.e. less than $3 \sigma$ ) in our measurements. 9 objects of this kind ( 3 of which are more than $2 \sigma$ above the normality line) may be grouped the following way:
One cool CP2 star: HR 1217, a SrCrEu star with $b-y=$ 0.186 is well beyond the limit where the $\Delta a$-index detects most of the CP2-stars (see Maitzen 1976).

Four mild or marginal CP2/CP4 stars: HR $280=\alpha \mathrm{Scl}$ was included in Maitzen's (1981) study of He-weak stars where he observed photometric peculiarity with $\Delta a=$ 0.018, while we obtain a bit less: 0.012 .

HR $1541=$ HD 30612 is B8 II/III (p Si) according to Michigan I. In very good accord with the present value (0.010) Maitzen (1980) obtained two $\Delta a$-values, 0.012 and 0.013 , just at the threshold limit of peculiarity in his photometry. Contrary to many other cases where giant luminosity erroneously obtains instead of Silicon peculiarity, it results here from existing Stroemgren-Crawford photometry (see Hauck \& Mermilliod 1980) together with peculiarity both in spectroscopy and photometry.

HR $2761=$ HD 56455 has been classified as Silicon star both in the BS and the Michigan II catalogues. Support for the CP2-type came from different studies of photometric variability (for references see Catalano \& Leone 1993) exhibiting a period of about 2 days and decreasing amplitude from the near ultraviolet to the yellow region. Taking Geneva photometry (Waelkens 1985), more precisely the $\Delta(V 1-G)$-index we find that this star varies between -0.006 (= normal star) and +0.014 (=peculiar star). Obviously our $\Delta a=0.007 \mathrm{mag}$ corresponds to an intermediate phase.

HR $2971=$ HD 61966 has been detected in the Michigan survey (MS II), but the type given indicates the marginal nature of its peculiarity: B9 IV/V (p Si). Our $\Delta a=0.005$ and the Geneva $\Delta(V 1-G)=-0.005$ coincide in photometric non-peculiarity. One should reinvestigate 
this star by spectroscopy in order to have a closer look on possibly existing traces of peculiarity.

Three stars with controversial spectroscopic peculiarity: HR $2863=\mathrm{HD} 59256$ has $\mathrm{B} 9 \mathrm{~V}$ (p Si) according to Michigan III, similar to the first assignment of Silicon peculiarity by Jaschek \& Jaschek (1960). Brandi \& Clariá (1973), however, reclassified this star pointing out that the Silicon features $4128-30$ and $3856-62$ were weak or absent, resp., but that the K-line was strong. Luminosity class V is contradicted by both Geneva and StroemgrenCrawford photometry which clearly indicate giant type. Our marginal $\Delta a$-value and the non-peculiar Geneva indices cast heavy doubt on whether this is a usual Silicon CP2-star.

HR $3001=$ NGC 2451-W233 has only one peculiarity classification: B9VpSi in Feinstein (1966). Hiltner et al. (1969) classify B7III, as well as Levato \& Malaroda (1975), and Michigan III gives B8IV. Our nonpeculiar $\Delta a=0.001 \mathrm{mag}$ is corroborated by Maitzen \& Catalano (1986) who obtained $\Delta a=0.003 \mathrm{mag}$, and also the Geneva system peculiarity parameter $\Delta(V 1-G)=-0.006$ (Hauck $\&$ North 1993) points to a normal star. There is only one source which might indicate peculiarity, i.e. Nissen (1974) who found He-weakness at the $3 \sigma$ limit for HR 3001 and a surface gravity typical for luminosity class V. Taking all available information together it can be assumed that this star is He-weak, but very probably belonging to the nonmagnetic branch of CP4-stars.

HR $3151=$ HD 66255 is a supergiant according to Michigan II (B9 Ib), while Jaschek \& Jaschek (1959) classify A0p Si using relatively high dispersion (42 A/mm). Brandi \& Clariá (1973) reclassify this star and note that "Silicon is very weak". Hauck \& North (1982) obtained $\Delta(V 1-G)=0.000$ which is non-peculiar and matches well our low $\Delta a=0.005$ mag. Both Geneva and Stroemgren photometry indicate giant, not supergiant luminosity. This star is variable with a typical CP2-period length $(P=6.81780$ days, Catalano \& Leone 1993). On the other hand its longitudinal magnetic field as measured by Bohlender et al. (1993) is only $1 \sigma$ below zero. We conclude that the majority of observational evidence is not in favour of a CP2-star, but that it deserves spectroscopic reassessment.

$A$ special case: HR $2875=\mathrm{HD} 59635$ has $\Delta a=0.012 \mathrm{mag}$, close to the $3 \sigma$ level. BS has it as B5Vp, but Michigan III as B3V. The former comes from Hiltner et al. (1969) who note abnormally strong Silicon lines. Buscombe \& Morris (1958) classify B3III and obtain radial velocity variability. From Rufener (1988) we calculate class V luminosity and peculiarity parameters below the $2 \sigma$ level. This object could be a pronounced Silicon variable and should be studied in more detail. For the very early spectral type we list it as CP4 object.

\subsection{Comparison of photometric and spectroscopic peculiarity detection performances}

Using for $\sigma$ the average scatter 4.74 mmags about the normality line for all normal stars (Sect. 2.3) our $\Delta a$ photometry yields 30 peculiarity candidates which are above the $+3 \sigma$ line in Fig. 2. But in Sect. 2.3 we also stated that the scatter of the hotter stars is slightly smaller than the average scatter and that for them we therefore have to use 13.5 mmags as $3 \sigma$ level. This way we obtain three further peculiar stars (HR 1100, HR 2424 and HR 3413). No star of the cooler division mentioned in Sect. 2.3 is lost from the list of photometric peculiar objects by applying the larger $3 \sigma$ threshold determined for them. Thus, we arrive finally at 33 objects which have peculiar positive $\Delta a$-values ( $=$ deviations above the normality line).

One of them is a CP1 (Am) star (HR 1730) and 2 are A and F supergiants (HR 2785 and 2874) according to spectroscopic sources. The degree of impurity for the photometric detection of magnetic peculiar stars in the $\Delta a$-system is therefore in our sample 3 out of 33 objects $(9.1 \%)$.

Statistically this impurity contribution may be considered to be counterbalanced by those stars of our sample, which have been classified as peculiar by spectroscopy, but escaped detection in our photometric system. From the discussion in the preceding section four stars emerged with spectroscopic peculiarity classifications which can be regarded as well established: HR 1217 (at the cool end of the CP2 domain), and the hot stars HR 280, 1541 and 2761 which were previously shown to be photometrically peculiar. With some probability also HR 2875 could join this group exhibiting a $\Delta a$-value (12 mmags) which is above the $2 \sigma$ level. The 4 (maybe 5 ) non-detections of CP2/4 stars by our actual photometry are nearly compensated statistically by the impurity fraction mentioned before. Hence, altogether, we arrive at 34 (maybe 35) magnetically peculiar stars from the photometric side.

The situation with spectroscopy is as follows: Our sample contains 34 stars with CP2 classification. In addition, there are 10 Helium abnormal stars which we denote as CP4. Therefore, both He-weak and He-strong stars appear here under this label. Both groups seem to have a magnetic and a non-magnetic branch. From all those CP4 stars only a fraction of the He-weak subgroup has been shown by Maitzen (1984) to exhibit peculiarity in $\Delta a$ as members of the magnetic branch. In the present study only two CP4-objects show up as such, and we therefore arrive at 36 CP2/4 stars to be considered as number of magnetically peculiar stars based on spectroscopy. Again applying the concept of balance between impurity and misses we notice:

Three stars (HR 2863, 3001 and 3151) with controversial peculiarity classifications and HR 2971 which may be only very marginally peculiar, are photometrically normal and seem to be poorly related to the magnetic CP-stars if at all and should therefore be considered as impurity 
fraction of spectroscopic detection. On the other hand there are 2 very probable misses of peculiarity (HR 1973 and 3203$)$ by spectroscopic classification.

Considering this balance we should retain 34 well established CP2/4 magnetic stars after consideration of the spectroscopic detection technique.

Summarizing we find 34 objects which can be considered as reliable members of the group of magnetic peculiar stars in the present sample.

Comparing the raw numbers of both techniques we notice a slight preponderance of spectroscopic detections, which even becomes stronger if we include also the cases of controversial peculiarity classifications. But this difference can be safely explained by the fact that photometry (i.e. measurements of the $\lambda 5200$ feature) is free of subjective assessments while spectroscopic classification is a highly complex human pattern recognition process in which e.g. expectation behaviour may favour (in some cases) the detection of peculiarity.

\subsection{Percentage of magnetic peculiar stars}

For a discussion of the frequency of $\mathrm{CP} 2 / \mathrm{CP} 4$ stars among the entire sample of normal and peculiar stars, not only as a single quantity, but distributed over the whole photometric interval we have plotted Fig. 6 . It shows the histograms of all stars of our sample for both their intrinsic $B-V$ and $b-y$ values, resp., and also for the CP2 and CP1 stars. We have to notice that both in absolute numbers and in percent values the majority of CP2-stars (10.7\%) appear in the hottest bin: $-0.19 \leq B-V<$ -0.10 . This is not in perfect agreement with the results of Wolff (1968), who used a somewhat complementary sample (stars brighter than $V=5.0 \mathrm{mag}$ and in the northern hemisphere), since she obtained a maximum of CP2-stars $(14 \%)$ in the range $-0.09 \leq B-V<0.0$. One may speculate about the reason for this discrepancy both on the ground of her significantly smaller sample and a possible local feature of the spatial CP2 distribution, since her magnitude limit was rather restrictive. However, Wolff's (1968) percentages for the cool Ap stars (excluding Am stars) fully agree with our results: for the earlier group $5 \%(0.0 \leq B-V<0.1)$ and only marginally more than $1 \%$ for the remainder.

Considering $b-y$-values we obtain a rather similar picture: $9 \%$ are in the hottest group $(-0.100 \leq b-y<$ $-0.050), 6 \%$ in the following bin with negative $b-y$ values, while only $3 \%$ are peculiar in the range $0.0 \leq b-y<0.100$.

A comment is due on the shape of the histograms presented: While our sample of CP1-stars shows a very similar distribution for $B-V$ and $b-y$, the resemblance for our CP2-stars is less striking. There is a slight preponderance of negative $b-y$ values ( 3 objects) compared to negative $B-V$ values which is to be expected from the differential blueing effect on the Stroemgren index $b-y$ in comparison to $B-V$ since $b$ is less affected by line absorption than $B$ and $y$ is more influenced by the $\lambda 5200$ absorption feature than $V$. On the other hand it is astonishing that the negative values of $B-V$ assemble with great majority in the hotter bin. But we have also to take into account that the relatively low number of CP2-stars and the corresponding need for big bin sizes are able to contribute to the actual picture.

Introducing spectroscopic results into this discussion we have to mention Abt (1979) who compared the number of Silicon stars brighter than $V=6$ mag in Osawa's (1965) list with the number of B5-A0IV and V stars in the BS catalogue of 1964 in the same magnitude and declination ranges and derived $6.5 \pm 2.4 \%$.

Despite the relatively large error this value (which is rather close to our result) is clearly smaller than the average frequency of CP2 stars with negative $B-V(10 \%)$ as derived from the study of Wolff (1968). Again it may be argued that this is the result of surveying a much larger volume than that used for the sample of Wolff. But there may be another or an additional explanation for this discrepancy: Abt (1979) obtains $5.4 \% \pm 2.3$ for the frequency of cool field CP2-stars. Even with the rather large error this result (i.e. nearly equal frequencies of hot and cool peculiar stars) can hardly be reconciled with the findings of Young \& Martin (1973) and North (1993) - the latter obtained a frequency of about $10 \%$ for the Silicon stars and about $4 \%$ for the cool CP2 stars, both in open clusters and the field. A rather straightforward explanation for this difference is the discrepant definition of the borderline between hot and cool Ap-stars which caused an increase of cool CP2 stars at the expense of the hot CP2 stars in the case of Abt (1979). The reversed situation seems to apply for the photometric definition of the borderline. Setting it to zero in both indices $B-V$ and $b-y$ we do transfer a number of objects recognized spectroscopically as cool Ap-stars to the domain of hot stars, just because of their bluer colors.

Confirmation for this argument comes from a comparison of the hot/cool CP2-star ratios of our sample. With the photometric criterion (negative/positive colour indices) we obtain $4: 1$, while with the spectroscopic division (Silicon and He wk/all other CP2 stars) the ratio is close to $3: 1$. Since the ratio for the normal stars with analogue criteria is roughly $2: 1$, the frequency ratios are 2 for spectroscopy and 1.5 for photometry.

With these considerations it seems that the discrepancy between spectroscopic and photometric frequencies has been removed to a large degree, especially if one takes into account that the the identification of Silicon stars suffers from the ambiguous interpretation of enhanced Silicon line strengths which may also be caused by higher luminosity of a normal star. 

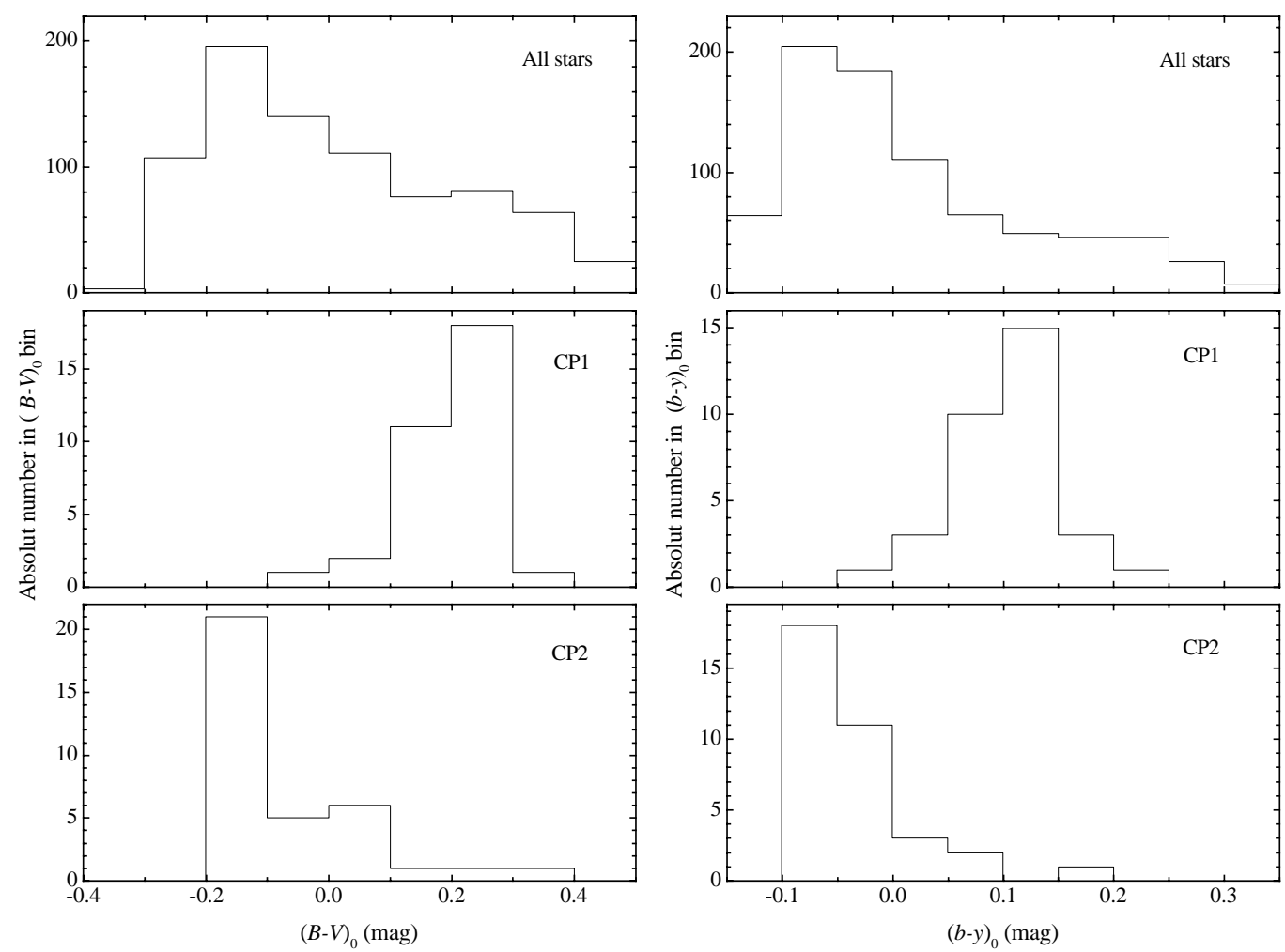

Fig. 6. Distribution of intrinsic $B-V$ and $b-y$ all stars, for the CP2 and CP1 objects

3.5. Behaviour of other stars not used for the derivation of the normality line

These stars are contained in Fig. 5 together with our main targets, the magnetic peculiar stars. By different symbols the following groups are represented there:

\subsection{1. $\lambda$ Bootis stars}

This type of peculiar stars has been shown to exhibit negative $\Delta a$ values by Maitzen \& Pavlovski (1989). Five stars of this group in our sample (HR 12, 541, 1570, 2039, 3651) have also been observed by those authors and show the same tendency of slightly negative $\Delta a$ values. For our 9 $\lambda$ Boo objects we obtain a mean $\Delta a=-0.0087(0.0050$ s.d.) which is nearly two $\sigma$ of the normal stars below the normality line.

\subsubsection{Be/shell-stars}

Their mean $\Delta a=-0.0025$ (0.0060 s.d.) for 25 objects. Since objects in the emission phase tend to lie below the normality line ( 2 objects with $3 \sigma$ ), whereas during the shell episodes $\Delta a$ becomes mildly positive (in the extreme case of Pleione even substantially peculiar, as shown by Maitzen \& Pavlovski 1987), we interpret the marginal negative average of $\Delta a$ as result of mutual compensation of both effects. It is comforting that this investigation did not yield another Pleione-like shell star with contaminatingly large $\Delta a$. This means that the B-type part of the CP2domain is practically free of any effects causing positive $\Delta a$ deviations other than that of magnetically peculiar stars.

\subsubsection{Am (CP1) and $\delta$ Del stars}

The 33 metallic line stars are statistically above the normality line by $\Delta a=0.0054$ (0.0053 s.d.). The two CP1stars with largest $\Delta a$ are even at the $3 \sigma$ level as mentioned above contributing to some slight impurity in the detection of CP2 stars. The $\delta$ Del stars (7 objects) show up with nearly the same average positive $\Delta a=0.0050$ (0.0063 s.d.) and are the continuation of the CP1-stars to the red with some overlap in our diagram.

\subsubsection{HgMn (CP3) stars}

Our 15 objects of this group are slightly above the normality line with an average $\Delta a=0.0041$ (0.0050 s.d.). Though no member of this group reaches $3 \sigma$ in this paper one should be aware that extreme cases (transition objects to CP2?) may surpass this limit, especially when higher precision (more measurements per star) should be available. 


\subsubsection{He-abnormal (CP4) stars}

The average value for 9 stars of this group is $\Delta a=0.0071$ (0.0078 s.d.). This, however, is the result of mixing together He-strong stars, which are among the hottest stars of our sample and line up very nicely close to the normality line (irrespective whether they are magnetic or not), and He-weak stars discussed above, which show partially peculiar $\Delta a$-values related to the presence of magnetic fields of the CP2 kind.

\subsubsection{Double stars}

Figure 7 shows the diagram $a$ vs. $b-y$ for those 170 double stars which had been excluded either as spectroscopic binaries or as close visual doubles with low magnitude differences as described before. The average $\Delta a=-0.0002$ and the average scatter 0.0050 around the normality line express that we were too cautious eliminating these stars when calculating the normality line. Nevertheless, it is interesting to note a slight preponderance of positive $\Delta a$ values among the late $\mathrm{A}$-type stars which is reminiscent of the behaviour of Am- and $\delta$ Del stars.

\subsection{Conclusions}

The following conclusions can be drawn:

1. Statistically $\Delta a$ photometry yields as many peculiar objects related to the magnetic branch as spectroscopy based on classification dispersion. Both techniques suffer from some contaminating effects which concern, however, only about 10 percent of the sample: the main reason for spectroscopy is the discrepancy in the interpretation of enhanced Silicon lines as indicators for luminosity or chemical peculiarity. This may lead to both erroneous inclusions of CP-stars and misses which we have shown to occur in literature. Photometry of the $\lambda 5200$ feature, in our case $\Delta a$-photometry, turned out to be slightly contaminated in the red domain of our sample by moderate size $\Delta a$-excesses of CP1 and AF-supergiant stars, quantitatively compensated by misses of mild CP2-stars part of which are caused by variability of $\Delta a$.

2. Within the $3 \sigma$ level there are statistical deviations of other groups of stars which are confirmations of earlier findings: CP1 stars slightly above the normality line, as well as CP3 and part of the He abnormal stars (the He weak stars), while emission line B-stars and $\lambda$ Boo stars are located below it. No Pleione-like shell star was found, i.e. with peculiar $\Delta a$. New findings are statistically positive deviations of $\delta$ Del and AF supergiant stars.

3. Nonpeculiar visual double stars and spectroscopic binaries do not significantly alter the location of the normality line as well as the scatter around it.
4. Histograms of the intrinsic $B-V$ and $b-y$ values of the CP2-stars and all stars of our sample show an average maximum of $10 \%$ for the fraction of the bluest peculiar stars (those with $-.19 \leq B-V<-.10$ or $-.100 \leq$ $b-y<-.050)$ among all stars of our sample with the same colours. In the colour ranges $0.0 \leq B-V<0.20$ and in $0.0 \leq b-y<0.100)$ the average frequency of CP2-stars has come down to $3 \%$.

5. The results of our study are encouraging for larger statistical studies using CCD-photometry in the $\Delta a$ system especially in the case of volume-limited samples, e.g. galactic clusters, also in order to solve the problem whether CP2-frequencies are similar to the field or not. The photometric detection will refer to those stars which would be also detectable by low (=classification) spectroscopy - at the expense of a prohibitive demand for enormous telescope time and classification efforts. Stars with lower degrees of peculiarity would be detectable only by high resolution spectroscopy restricted to very small samples. Doing statistics with photometrically detected chemically peculiar stars we have to assume that their numbers are also representative for the appearance of weakly peculiar stars spatially associated with them.

Acknowledgements. This work was supported by the Austrian Fonds zur Förderung der wissenschaftlichen Forschung under project number PHY-4715. We are indebted to ESO and its staff for providing excellent support and services.

\section{References}

Abt H.A., 1979, ApJ 230, 485

Abt H.A., Cardona O., 1983, ApJ 272, 182

Bidelman W.P., MacConnell D.J., 1973, AJ 78, 687

Brandi E., Clariá J.J., 1973, A\&AS 12, 79

Bohlender D.A., Landstreet J.D., Thompson I.B., 1993, A\&A 269,355

Buscombe W., 1980, MK Spectral Classifications 4th General Catalogue, Evanston

Buscombe W., Morris P.M., 1958, MNRAS 118, 609

Catalano F.A., Renson P., Leone F., 1991, A\&AS 87, 59

Catalano F.A., Leone F., 1993, A\&AS 97, 501

Curchod A., Hauck B., 1979, A\&AS 38, 449

Feinstein A., 1966, PASP 78, 301

Hauck B., 1974, A\&A 32, 447

Hauck B., Mermilliod M., 1980, A\&AS 40, 1

Hauck B., North P., 1982, A\&A 114, 23

Hauck B., North P., 1993, A\&A 269, 403

Hiltner W.A., Garrison R.F., Schild R.E., 1969, ApJ 157, 313

Hoffleit D., Jaschek C., 1982, The Bright Star Catalogue 4th Rev. Ed., Yale Univ. Obs., New Haven

Houk N., 1978, Michigan Catalogue of Two-Dimensional Spectral Types for the HD Stars Vol. 2, Univ. of Michigan, Ann Arbor

Houk N., 1982, Michigan Catalogue of Two-Dimensional Spectral Types for the HD Stars Vol. 3, Univ. of Michigan, Ann Arbor 


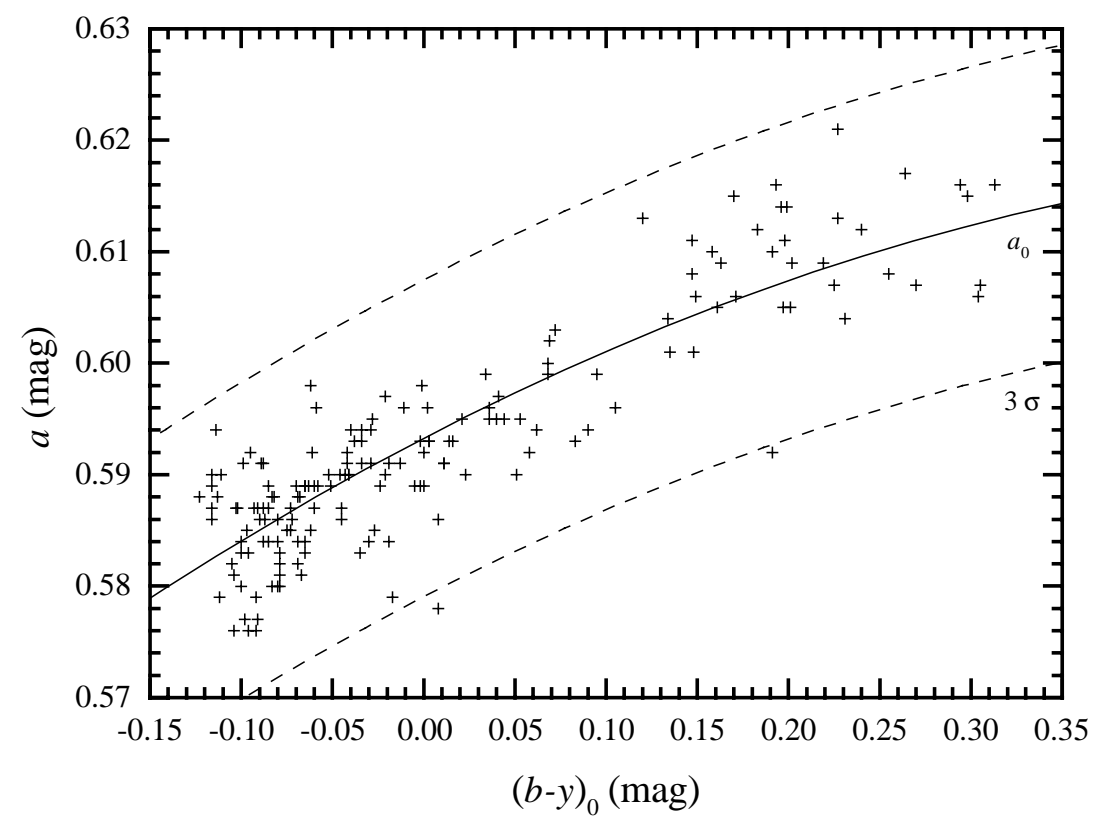

Fig. 7. $a$ versus $(b-y)_{0}$ diagram for double stars

Houk N., Cowley A.P., 1975, University of Michigan Catalogue of Two-Dimensional Spectral Types for the HD Stars Vol. 1, Univ. of Michigan, Ann Arbor

Houk N., Smith-Moore M., 1988, Michigan Catalogue of TwoDimensional Spectral Types for the HD Stars Vol. 4, Univ. of Michigan, Ann Arbor

Jaschek M., Jaschek C., 1959, PASP 71, 48

Jaschek M., Jaschek C., 1960, PASP 72, 500

Levato H., Malaroda S., 1975, PASP 87, 823

Lucke P.B., 1978, A\&A 64, 367

Maitzen H.M., 1976, A\&A 51, 223

Maitzen H.M., 1980, A\&A 84, L9

Maitzen H.M., 1981, A\&A 95, 213

Maitzen H.M., 1984, A\&A 138, 493

Maitzen H.M., Catalano F.A., 1986, A\&AS 66, 37

Maitzen H.M., Lebzelter Th., 1993, Inf. Bull. Var. Stars No. 3856

Maitzen H.M., Pavlovski K., 1987, A\&A 178, 313

Maitzen H.M., Pavlovski K., 1989, A\&AS 81, 335

Maitzen H.M., Pavlovski K., 1997 (in preparation)

Maitzen H.M., Vogt N., 1983, A\&A 123, 48

Nissen P.E., 1974, A\&A 36, 57

North P., 1993, in: Peculiar Versus Normal Phenomena in AType and Related Stars, Dworetsky M.M., Castelli F. and Faraggiana R. (eds.), ASP Conf. Ser. 44, p. 577

Osawa K., 1965, Ann. Tokyo Astron. Obs. Ser. 2, 9, 123

Rufener F., 1988, Catalogue of Stars measured in the Geneva Observatory Photometric System (4th ed.), Obs. de Genève, Sauverny

Waelkens C., 1985, A\&AS 61, 127

Wolff S.C., 1968, PASP 80, 281

Young A., Martin A.E., 1973, ApJ 181, 805

\section{Appendix}

In the following both the observing $\log$ (Table 1) and the photometric data (Table 2) are listed.
Table 1 gives the numbers of the runs, starting and end dates, the total number of nights and the observers. Their abreviations B, F, P, R, and V stand for Blest, FaúndezAbans, Perez, Roman, and Vogt, respectively.

Table 1. Observing log

\begin{tabular}{cclrlrcl}
\hline run & year & \multicolumn{2}{c}{ start } & \multicolumn{2}{c}{ end } & nights & observer \\
\hline 1 & 1974 & Sep. & 20 & Sep. & 29 & 6 & V \\
2 & 1974 & Oct. & 19 & Nov. & 4 & 11 & B, P, V \\
3 & 1974 & Nov. & 14 & Dec. & 1 & 5 & R \\
4 & 1975 & Mar. & 27 & Mar. & 31 & 4 & V \\
5 & 1975 & May & 27 & May & 28 & 2 & V \\
6 & 1975 & Sep. & 23 & Oct. & 1 & 7 & V, F \\
7 & 1975 & Nov. & 21 & Dec. & 4 & 11 & F, V \\
8 & 1976 & Jan. & 2 & Jan. & 7 & 5 & F \\
9 & 1976 & Mar. & 21 & Mar. & 25 & 5 & F \\
10 & 1976 & Jun. & 28 & Jun. & 30 & 3 & F \\
\hline
\end{tabular}

Table 2 on the following pages lists the HR and HD numbers, the run (see Table 1) in which the observations were obtained (sometimes several observations per run and/or on several runs), $a, \Delta a$ absolute and expressed in units of the average standard deviation of normal stars from the normality line, $b-y$ and its dereddened quantity $(b-y)_{0}, m_{1}$, the spectral types from the BS Catalogue and the Michigan spectral survey (where available), then a column which indicates whether the object was used for the construction of the normality line ("N") or entered the group of double stars ("D"), and finally the peculiarity group to which the star belongs (Preston's CP1-4, $\lambda$ Boo, $\delta$ Del, emission line objects "E", supergiants "SG" and a Barium star "Ba"). 